\title{
Optical domain service differentiation using spectral-amplitude-coding
}

\begin{abstract}
We have implemented a new service differentiation technique in the optical domain using a spectral-amplitude-coding (SAC) variant of optical code division multiple access (OCDMA). The newly developed code, named KS (Khazani-Syed) is compared mathematically with other codes which use similar techniques. In our proposal, multiple weights are used to support 'triple-play' services (audio, video and data) with different quality-of-service (QoS) requirements. The results characterizing the bit-error-rate (BER) with respect to the total number of active users show that KS offers a significantly improved performance over the previous reported techniques by accommodating additional 30 users with shorter code length and smaller code weight at BER of 10-9. In variable weight system, we have shown that KS codes with larger weight always have the best performance when other users of different weights are present in the system.
\end{abstract}

Keyword: Optical CDMA; Spectral-amplitude-coding; Variable weight system 